\title{
Knee replacement outcome predicted by physiotherapists: a prospective cohort study
}

\author{
Marius Henriksen $^{\text {Corresp., } 1}$, Hiwa Mukriyani ${ }^{1}$, Carsten Juhl ${ }^{2,3}$ \\ ${ }^{1}$ The Parker Institute, Copenhagen University Hospital Bispebjerg-Frederiksberg, Copenhagen, Denmark \\ Department of Physiotherapy and Occupational Therapy, Copenhagen University Hospital, Herlev-Gentofte, Copenhagen, Denmark \\ 3 Department of Sports Science and Clinical Biomechanics, University of Southern Denmark, Odense, Denmark \\ Corresponding Author: Marius Henriksen \\ Email address: marius.henriksen@regionh.dk
}

Background: Knee arthroplasty (KA) is commonly used for osteoarthritis of the knee joint and it is a highly successful procedure. Still, KA leaves $20 \%$ of patients dissatisfied with their outcome. The purpose of this study was to determine if a prognosis made by physiotherapists at the orthopaedic wards during the first post-operative days could predict the 6- and 12-months outcome of KA.

Methods: Physiotherapists at two orthopaedic wards in Denmark were asked to predict the 6- and 12months outcome of the KA patients they have treated post-operatively on a 0-10 scale (10 representing the best prognosis). At 6 and 12 months post-operatively the patients answered the Oxford Knee Score (OKS), EuroQol 5D-3L and Patient Acceptable Symptom State (PASS). Multivariable logistic regression analyses were performed to assess the prediction of PASS and treatment success. We assessed predictive performance by examining measures of calibration and discrimination.

Results: 361 patients were included. The models for PASS and Treatment Success showed poor to acceptable discriminative values (OR between 1.47 and 1.92 and areas under the curves of 0.62-0.73), however the calibration plots indicated significant uncertainties in the prediction.

Conclusion: Physiotherapists prognoses of recovery after KA are associated with 6- and 12-months patient reported outcomes and satisfaction but have weak predictive value. This study suggests that physiotherapists' prognoses may be useful as an additional source of information when identifying patients in need of additional post-operative care. 


\section{KNEE REPLACEMENT OUTCOME PREDICTED BY PHYSIOTHERAPISTS: A \\ 2 PROSPECTIVE COHORT STUDY}

3

4 Marius Henriksen ${ }^{1}$, Hiwa Mukriyani ${ }^{1}$, Carsten Juhl ${ }^{2,3}$

5

$6{ }^{1}$ The Parker Institute, Copenhagen University Hospital Bispebjerg-Frederiksberg, Copenhagen,

7 Denmark

82 Department of Physiotherapy and Occupational Therapy, Copenhagen University Hospital,

9 Herlev and Gentofte, Copenhagen, Denmark

$10{ }^{3}$ Department of Sports Science and Clinical Biomechanics, University of Southern Denmark,

11 Odense, Denmark

12

13 Corresponding Author:

14 Marius Henriksen ${ }^{1}$

15 The Parker Institute, Bispebjerg-Frederiksberg Hospital, Ndr. Fasanvej 57, DK-2000

16 Frederiksberg, Denmark

17 Email address: Marius.henriksen@regionh.dk 
19 Abstract

20 Background: Knee arthroplasty (KA) is commonly used for osteoarthritis of the knee joint and

21 it is a highly successful procedure. Still, KA leaves $20 \%$ of patients dissatisfied with their

22 outcome. The purpose of this study was to determine if a prognosis made by physiotherapists at

23 the orthopaedic wards during the first post-operative days could predict the 6- and 12-months

24 outcome of KA.

25 Methods: Physiotherapists at two orthopaedic wards in Denmark were asked to predict the 6-

26 and 12-months outcome of the KA patients they have treated post-operatively on a $0-10$ scale (10

27 representing the best prognosis). At 6 and 12 months post-operatively the patients answered the

28 Oxford Knee Score (OKS), EuroQol 5D-3L and Patient Acceptable Symptom State (PASS).

29 Multivariable logistic regression analyses were performed to assess the prediction of PASS and

30 treatment success. We assessed predictive performance by examining measures of calibration

31 and discrimination.

32 Results: 361 patients were included. The models for PASS and Treatment Success showed poor

33 to acceptable discriminative values (OR between 1.47 and 1.92 and areas under the curves of

34 0.62-0.73), however the calibration plots indicated significant uncertainties in the prediction.

35 Conclusion: Physiotherapists prognoses of recovery after KA are associated with 6- and 12-

36 months patient reported outcomes and satisfaction but have weak predictive value. This study

37 suggests that physiotherapists' prognoses may be useful as an additional source of information

38 when identifying patients in need of additional post-operative care. 
40

41

42

43

44

45

46

47

48

49

50

51

52

53 54 is equally important.

55 Physiotherapists play an important role in the post-operative treatment of patients that have 56

57 58

\section{Introduction}

Knee arthroplasty (KA) is considered a successful orthopaedic procedure to alleviate knee pain and disability in knee osteoarthritis (OA) and the demand for KA is large and growing worldwide (Ackerman et al. 2019; Culliford et al. 2015; Nemes et al. 2015). Despite being considered a generally safe and successful procedure, there is a significant proportion (20\%) of patients who endure years of disability and dissatisfaction with their postoperative function (Bourne et al. 2010; Gunaratne et al. 2017; Kahlenberg et al. 2018). Many of these patients do not undergo revision surgery, but all add to the society's burden of health care as clinicians and allied services strive to remedy their dissatisfaction.

While efforts have been made to predict the best candidates for KA (Birch et al. 2019;

Harbourne et al. 2019; Judge et al. 2012) it has proven very difficult to identify robust preoperative prognostic factors (Gunaratne et al. 2017). It is important to be able to identify inappropriate candidates pre-operatively, but until that is possible, efforts to improve postoperative rehabilitation and identify individuals that may need special attention post-operatively

Physiotherapists play an important role in the post-operative treatment of patients that have received a KA. In general patients are mobilised very early, and rehabilitation is commenced during the first days after surgery (or even on the same day) while the patient is still hospitalised. There are numerous factors that can predict the outcome of KA, of which most are either poor predictors or unsatisfactorily assessed scientifically (Harmelink et al. 2017). In daily clinical physiotherapy practice at the orthopaedic wards, previous experiences, knowledge, and personal interaction with a patient altogether result in the physiotherapist's intuition about the future course of a patient's recovery. Previously, it has been shown that physiotherapists' prognoses 
63 during the initial clinical encounter for the projected outcome of patients with low back and neck

64 pain they treated, was associated with the actual clinical outcome (Cook et al. 2015). However, it

65 is unknown if such intuition is a reliable predictor of the outcome of KA.

66 With this study we aimed to assess if physiotherapists treating inpatients at orthopaedic wards

67 could predict the 6- and 12-months post-operative self-reported pain, functional status and health 68 related quality of life of patients undergoing KA.

69

\section{Materials \& Methods}

71 The study is a prospective, pragmatic, longitudinal cohort study conducted from December 2016

72 to December 2019 with a 6- and 12-month follow-up. The study was conducted according to a

73 prespecified protocol, which was pre-registered and submitted to the Health Research Ethics

74 Committee of The Capital Region of Denmark (file number: 16039254). The committee deemed

75 the study exempt from approval as the study only uses questionnaire data. Such studies can be

76 implemented without approval from the Health Research Ethics Committee according to Danish

77 legislation. The prespecified study protocol was pre-registered at www.clinicaltrials.gov

78 (Identifier: NCT02982785) prior to data collection.

79

80 Participants

81 Participants were recruited from the orthopaedic wards at Bispebjerg-Frederiksberg Hospital and

82 Herlev-Gentofte Hospital in Copenhagen, Denmark. The inclusion criteria were: Primary total

83 KA for knee OA; Age >18 years; Read and speak Danish and having an email-address. The

84 exclusion criteria were: Revision surgery and cognitive or mental conditions precluding reliable 
85 answers to online questionnaires (determined either from medical records or judged by the

86 including physiotherapists). All participating patients signed an informed consent.

87

\section{Procedures}

89 During the post-operative hospital stay, eligible participants were identified by the

90 physiotherapists and invited to participate in the study. Upon signed informed consent, baseline

91 data were collected using standardized forms. At discharge, physiotherapists delivering the initial

92 in-patient physiotherapy and mobilization judged the participants' prognosis (see below).

93 Study data were collected and managed using REDCap electronic data capture tools hosted at

94 The Capital Region of Denmark (Harris et al. 2019; Harris et al. 2009). REDCap (Research

95 Electronic Data Capture) is a secure, web-based software platform designed to support data

96 capture for research studies, providing 1) an intuitive interface for validated data capture; 2)

97 audit trails for tracking data manipulation and export procedures; 3) automated export procedures

98 for seamless data downloads to common statistical packages; and 4) procedures for data

99 integration and interoperability with external sources. Via REDCap, individual internet-

100 hyperlinks were emailed to the participants 6 and 12 months after discharge. The hyperlinks led

101 to a secured webpage on which the patients answered questionnaires. The participant-submitted

102 responses were automatically registered in a secured database. At both 6- and 12-months data

103 collection points an email reminder was sent to participants if they did not answer the

104 questionnaires within 7 days.

105

106 Physiotherapists' prognoses 
107 The physiotherapists estimated each patient's potential for a successful outcome after 6-12

108 months at discharge based on professional appraisal. The physiotherapists were asked to appraise

109 all parts of their evaluation in their prognosis of each patient. The physiotherapists were

110 instructed to score each patient on a 1-10 Likert scale (1 representing a very poor projected

111 outcome, 10 representing an excellent projected outcome). The physiotherapists scored each

112 patient following their complete encounter with the patient. This included the physiotherapist's

113 assessment of the patient's resources (personal, material, social, etc.), personality, medical

114 history, comorbidities, surgery reports, physical examination(s), in-patient physiotherapy

115 treatment response(s), physiotherapeutic (re)assessments, and more. The prognosis was not 116 disclosed to the participants.

\section{Outcomes}

119 The primary outcome measure was the Oxford Knee Score (OKS). The Oxford Knee Score

120 (OKS) is a 12-item Patient Reported Outcome questionnaire developed specifically to assess the

121 patient's perspective of outcome following KA (Dawson et al. 1998). Standardized answer

122 options are given (5 Likert boxes) and each question is assigned a score from 0 to 4 . A total score

123 is calculated that ranges from 0 and 48, with 48 indicating the best outcome. The OKS is short,

124 practical, reliable, valid, and sensitive to clinically important changes over time (Dawson et al.

125 1998). The OKS scores were dichotomised using an established cut-off value for treatment

126 success after TKA of 32.5 points (Hamilton et al. 2018). The dichotomised OKS was labelled as

127 'Treatment Success' for scores above 32.5 at both 6- and 12-months follow-up.

128 In addition to the single OKS summary score, we also calculated the OKS pain and OKS

129 function sub-scale scores that were standardized to a range from 0 (worst) to 100 (best). 
130 Health outcome and quality of life was assessed using the European Quality of Life (EuroQoL)

131 questionnaire (EQ-5D-3L). EQ-5D-3L is a standardized patient-reported instrument for use

132 measuring health outcome and quality of life (EuroQol 1990). EQ-5D-3L is designed for self-

133 completion by respondents and is ideally suited for use in surveys.

134 The EQ-5D-3L consists of a descriptive system and a Visual Analogue scale (EQ-5D-VAS). The

135 descriptive system comprises 5 dimensions (mobility, self-care, usual activities, pain/discomfort, 136 and anxiety/depression). Standardized answer options are given (3 Likert boxes) and each

137 question is assigned a score from 1 to 3 . From the answers an EQ-5D-3L index score is

138 calculated based on Danish normative equations. The index ranges from - -0.624 (worst) to 1.000

139 (best). The EQ-5D-VAS records the respondent's current self-rated health on a $10 \mathrm{~cm}$ Visual

140 Analogue Scale (VAS) with endpoints labelled 'the best health you can imagine' and 'the worst

141 health you can imagine'.

142 We also applied a single question regarding the Patient Acceptable Symptom State (PASS)

143 (Tubach et al. 2005) to assess the patient's satisfaction with their state of symptoms at 6 and 12

144 months after surgery. PASS is assessed as a dichotomous outcome (yes/no) to the question:

145 "Considering your knee function, do you feel that your current state is satisfactory? With knee

146 function you should take into account all activities you have during your daily life,

147 Sport/Recreational activities, your level of pain and other symptoms, and your knee related $148 Q O L "$

\section{Statistical analyses}

150 The 6- and 12-months follow-up data were analyzed separately. The associations between the 151 physiotherapists' prognostic scores and the dichotomous PASS and Treatment Success variables 
152 were assessed using logistic regression analyses with the physiotherapists' prognostic scores as

153 independent variable and PASS ('yes') and Treatment Success as dependent variables.

154 The predictive performance of the physiotherapists' prognostic scores was analysed using

155 calibration and discrimination measures. Discrimination relates to the ability of the model to

156 discriminate between patients who have answered ' $y e s$ ' to the PASS question or achieved

157 Treatment Success from those who have answered 'no' or did not achieve Treatment Success.

158 These were evaluated by calculating the areas under the receiver operating characteristic curves

159 (AUC) and Nagelkerke $\mathrm{R}^{2}$ as an indication of explained variation. Calibration relates to the

160 agreement between the projected and observed outcome and was evaluated by means of

161 calibration plots, in which patients were classified by the predicted risk of the observed

162 prognostic scores, supplemented by loess lines over the predicted probability range. The loess

163 line of a prefect prediction model lies on the $45^{\circ}$ slope for agreement with the observed outcome.

164 The associations between the physiotherapists' prognostic scores and the continuous variables

165 OKS, OKS-pain, OKS-function, EQ-5D-3L index and EQ-5D-VAS were assessed by univariate

166 linear regression analyses with the physiotherapists' prognostic scores as independent variable

167 and OKS, OKS-pain, OKS-function, EQ-5D-3L and EQ-5D-VAS as dependent variables. The

168 associations were assessed via the estimated slopes (beta) and explained variance $\left(\mathrm{R}^{2}\right)$.

169 P-values of $<0.05$ were considered statistically significant.

170 


\section{Results}

172 From December 2017 to December 2018 a total of 382 individuals (57\% women) were invited to 173 participate (Table 1). Of these 20 were not eligible and 1 did not have a prognostic score.

174 Accordingly, 361 patients were included with a mean age of 69.2 years (SD 7.9). There were 42 175 patients who did not respond to any survey or reminders, 16 who responded to the 6-month but 176 not the 12-month survey, and 12 who did not respond to the 6-months but responded to the 12177 month survey (Figure 1). Thus, at the 6-month follow-up 307 answered the survey and at 12 178 months 303 answered the survey.

179

180 The prognostic scores ranged from 4 to 10, with a mean of 8.3 (SD 1.2) and the median being 9 181 (Table 1). Summaries of the outcome variables are presented in Table 2.

At the 6-month follow-up, the incidence of positive answers ('yes') to the PASS was 240 (78\%) and Treatment Success $(\mathrm{OKS}>32.5)$ was $251(82 \%)$. At the 12-month follow-up the incidence of positive PASS answer was 255 (85\%) and of Treatment Success was $251(83 \%)$.

A high prognostic score increased the odds of achieving PASS ('yes') at 6 months (OR $=1.47$ ( $95 \%$ confidence interval 1.19 to 1.82$)$ ) and at 12 months (OR $=1.45(95 \%$ CI 1.14 to 1.84$)$ ). Similarly, a high prognostic score increased the odds of Treatment Success at 6 months $(\mathrm{OR}=$ $1.62(95 \% 1.29$ to 2.03$))$ and at 12 months (OR $=1.92(95 \%$ CI 1.51 to 2.46$))$.

191

192 The performance of these models in terms of discrimination is presented in Table 3. The 193 prediction of positive answer to the PASS and Treatment Success at 6 months showed 
194 statistically significant yet poor discrimination (AUC 0.62 and 0.67 , respectively). At 12 months 195 the prediction of positive answer to the PASS and Treatment Success showed poor to acceptable 196 discrimination (AUC 0.64 and 0.73 , respectively).

197

198 The calibration of the models in the validation cohort is presented using calibration curves

199 (Figure 2) showing the performance of the models for PASS and Treatment Success at 6 and 12 200 months.

201

202 The results of the univariate regression analyses used to assess the prediction of the continuous

203 outcomes are given in Table 4 . The prognostic score was statistically significantly associated

204 with all the dependent variables at both 6 and 12 months, although with limited explained 205 variance $\left(\mathrm{R}^{2}<0.12\right.$; Table 4$)$. 


\section{Discussion}

208 In summary these findings support the hypothesis, that the prognostic scores of future outcomes 209 made by physiotherapists attending patients undergoing KA in the first days postoperatively 210 associate with the 6- and 12-month outcomes of KA. The discriminative performance can be 211 considered poor to acceptable. However, the AUC and ROC curve analyses did not suggest a 212 cut-off value that may be used to screen patients. Although the calibration plots suggest a linear 213 relationship between the predicted and observed probability of PASS and Treatment Success 214 there are significant uncertainties as judged by the $95 \%$ confidence intervals. From Figure 2 is 215 can be seen that the uncertainties (width of the 95\% CI) are related to the distribution of the 216 predicted probabilities. This is likely due to the small number of patients with low prognostic 217 scores (table 1). The linear regressions show that the physiotherapists' prognostic scores are 218 associated with the 6- and 12-months outcomes. Altogether the data suggest that the 219 physiotherapists' prognoses associate with the outcomes, but the predictive values of the scores 220 are not convincible. Thus, the physiotherapists' prognostic scores should be supported by other 221 information and individual assessments (such as age, sex, body weight, post-operative pain and 222 mobility, comorbidities etc.), when the future course of a patient is discussed and decisions about 223 possible extra attention is taken. Further studies on defining patients that may not have 224 successful outcomes of KA are needed.

225 The present study is the first on physiotherapists' prognoses of KA. Previously, it has been 226 shown that physiotherapists' prognoses during the initial clinical encounter for the projected 227 outcome of patients with low back and neck pain they treated, was associated with the actual 228 clinical outcome (Cook et al. 2015). 
229 A strength to the present study is the use of outcomes widely accepted for assessing outcomes of

230 KA (Conner-Spady et al. 2018; Dunbar et al. 2001; Garratt et al. 2004; Jin et al. 2019). The total

231 mean OKS score of the cohort, is comparable with the ones found in other studies (Williams et

232 al. 2013a; Williams et al. 2013b), which support the external validity of the results. Also, the

233 relatively large cohort provides some strength to the study. Further, the fact that the

234 physiotherapists who made the prognoses were not involved in delivery of any post-operative

235 rehabilitation is a significant strength as they could not influence the rehabilitation. Finally, it is a

236 strength that the study was carried out on two independent hospitals in Denmark, which

237 strengthen the generalizability of the results.

238 The study also has some limitations. Firstly, other types of information (such as pain intensity 239 and functional disability, body weight, comorbidities etc.) from the pre-operative and first post240 operative days could have been useful in identification of potentially modifiable factors (such as

241 body weight, muscle strength, functional disability, adjustment in treatment of comorbidities)

242 that associate with the prognoses. Such information could have helped identifying the underlying

243 factors that the physiotherapists based their prognoses on and used to propose future

244 interventions to mitigate negative outcomes. Further, the distribution of the prognostic scores

245 were skewed, with few low scores, which probably reflects the general successfulness of KA and 246 that the physiotherapists in general expect positive outcomes. Nevertheless, about $15 \%$ of the 247 patients did not reach PASS or Treatment Success after 12 months and could be candidates for 248 special post-operative attention . In this perspective, the physiotherapist prognosis may be used 249 to inform the identification of patients who may benefit from more intensive post-operative care 250 in order to enhance the outcome of KA. However, the present data suggest that such 251 identification should not rely solely on the physiotherapists prognoses but should incorporate 
252 other sources of information. Also, the instruments used to assess treatment success and patient

253 satisfaction (OKS and PASS) does not necessarily cover all aspects, as numerous factors

254 determine these. An OKS score above 32.5 (treatment success) does not necessarily indicate

255 success for the patient and the single yes/no PASS answer is not exhaustive. Several instruments

256 to assess patient satisfaction after KA exist (Kahlenberg et al. 2018), yet there is no consensus on

257 which instrument to use to assess treatment success or patient satisfaction. However, these

258 instruments (OKS and PASS) are widely used in OA and KA clinical and research settings.

259

260 Conclusions

261 In conclusion, physiotherapists prognoses of recovery after KA are associated with 6- and 12-

262 months patient reported outcomes and satisfaction but have weak predictive value. This study

263 suggests that physiotherapists' prognoses may be useful as an additional source of information

264 when identifying patients in need of additional post-operative care.

265

266

References

267 Ackerman IN, Bohensky MA, Zomer E, Tacey M, Gorelik A, Brand CA, and de Steiger R. 2019.

268 The projected burden of primary total knee and hip replacement for osteoarthritis in

269 Australia to the year 2030. BMC Musculoskelet Disord 20:90. 10.1186/s12891-019-2411-

270 9

271 Birch S, Stilling M, Mechlenburg I, and Hansen TB. 2019. The association between pain

272 catastrophizing, physical function and pain in a cohort of patients undergoing knee

273 arthroplasty. BMC Musculoskelet Disord 20:421. 10.1186/s12891-019-2787-6 
274 Bourne RB, Chesworth BM, Davis AM, Mahomed NN, and Charron KD. 2010. Patient

275

276

277

278

279

280

281

282

283

284

285

286

287

288

289

290

291

292

293

294 satisfaction after total knee arthroplasty: who is satisfied and who is not? Clin Orthop Relat Res 468:57-63. 10.1007/s11999-009-1119-9

Conner-Spady BL, Marshall DA, Bohm E, Dunbar MJ, and Noseworthy TW. 2018. Comparing the validity and responsiveness of the EQ-5D-5L to the Oxford hip and knee scores and SF-12 in osteoarthritis patients 1 year following total joint replacement. Qual Life Res 27:1311-1322. 10.1007/s11136-018-1808-5

Cook CE, Moore TJ, Learman K, Showalter C, and Snodgrass SJ. 2015. Can experienced physiotherapists identify which patients are likely to succeed with physical therapy treatment? Archives of Physiotherapy 5:3. 10.1186/s40945-015-0003-z

Culliford D, Maskell J, Judge A, Cooper C, Prieto-Alhambra D, Arden NK, and Group COS. 2015. Future projections of total hip and knee arthroplasty in the UK: results from the UK Clinical Practice Research Datalink. Osteoarthritis Cartilage 23:594-600. 10.1016/j.joca.2014.12.022

Dawson J, Fitzpatrick R, Murray D, and Carr A. 1998. Questionnaire on the perceptions of patients about total knee replacement. J Bone Joint Surg Br 80:63-69.

Dunbar MJ, Robertsson O, Ryd L, and Lidgren L. 2001. Appropriate questionnaires for knee arthroplasty. Results of a survey of 3600 patients from The Swedish Knee Arthroplasty Registry. J Bone Joint Surg Br 83:339-344. 10.1302/0301-620x.83b3.11134

EuroQol G. 1990. EuroQol--a new facility for the measurement of health-related quality of life. Health Policy 16:199-208. 10.1016/0168-8510(90)90421-9 
295 Garratt AM, Brealey S, Gillespie WJ, and Team DT. 2004. Patient-assessed health instruments 296 for the knee: a structured review. Rheumatology (Oxford) 43:1414-1423.

297 10.1093/rheumatology/keh362

298 Gunaratne R, Pratt DN, Banda J, Fick DP, Khan RJK, and Robertson BW. 2017. Patient 299 Dissatisfaction Following Total Knee Arthroplasty: A Systematic Review of the Literature. J Arthroplasty 32:3854-3860. 10.1016/j.arth.2017.07.021

301

302

303

304

305

306

307 308

309

310

311

312

313

314

315

316

Hamilton DF, Loth FL, MacDonald DJ, Giesinger K, Patton JT, Simpson AH, Howie CR, and Giesinger JM. 2018. Treatment Success Following Joint Arthroplasty: Defining Thresholds for the Oxford Hip and Knee Scores. J Arthroplasty 33:2392-2397. 10.1016/j.arth.2018.03.062

Harbourne AD, Sanchez-Santos MT, Arden NK, and Filbay SR. 2019. Predictors of return to desired activity 12 months following unicompartmental and total knee arthroplasty. Acta Orthop 90:74-80. 10.1080/17453674.2018.1542214

Harmelink KEM, Zeegers A, Hullegie W, Hoogeboom TJ, Nijhuis-van der Sanden MWG, and Staal JB. 2017. Are There Prognostic Factors for One-Year Outcome After Total Knee Arthroplasty? A Systematic Review. J Arthroplasty 32:3840-3853 e3841. 10.1016/j.arth.2017.07.011

Harris PA, Taylor R, Minor BL, Elliott V, Fernandez M, O'Neal L, McLeod L, Delacqua G, Delacqua F, Kirby J, Duda SN, and Consortium RE. 2019. The REDCap consortium: Building an international community of software platform partners. $J$ Biomed Inform 95:103208. 10.1016/j.jbi.2019.103208

Harris PA, Taylor R, Thielke R, Payne J, Gonzalez N, and Conde JG. 2009. Research electronic data capture (REDCap)--a metadata-driven methodology and workflow process for 
providing translational research informatics support. J Biomed Inform 42:377-381.

Jin X, Al Sayah F, Ohinmaa A, Marshall DA, and Johnson JA. 2019. Responsiveness of the EQ-

321

322

323

324

325

326

327

328

329

330

331

332

333

334

335

336

337

338 5D-3L and EQ-5D-5L in patients following total hip or knee replacement. Qual Life Res 28:2409-2417. 10.1007/s11136-019-02200-1

Judge A, Arden NK, Cooper C, Kassim Javaid M, Carr AJ, Field RE, and Dieppe PA. 2012. Predictors of outcomes of total knee replacement surgery. Rheumatology (Oxford) 51:1804-1813. 10.1093/rheumatology/kes075

Kahlenberg CA, Nwachukwu BU, McLawhorn AS, Cross MB, Cornell CN, and Padgett DE. 2018. Patient Satisfaction After Total Knee Replacement: A Systematic Review. HSS J 14:192-201. 10.1007/s11420-018-9614-8

Nemes S, Rolfson O, A WD, Garellick G, Sundberg M, Karrholm J, and Robertsson O. 2015. Historical view and future demand for knee arthroplasty in Sweden. Acta Orthop 86:426431. $10.3109 / 17453674.2015 .1034608$

Tubach F, Ravaud P, Baron G, Falissard B, Logeart I, Bellamy N, Bombardier C, Felson D, Hochberg M, van der Heijde D, and Dougados M. 2005. Evaluation of clinically relevant states in patient reported outcomes in knee and hip osteoarthritis: the patient acceptable symptom state. Ann Rheum Dis 64:34-37. 10.1136/ard.2004.023028

Williams DP, Blakey CM, Hadfield SG, Murray DW, Price AJ, and Field RE. 2013a. Long-term trends in the Oxford knee score following total knee replacement. Bone Joint J 95-B:4551. 10.1302/0301-620X.95B1.28573 
339 Williams DP, Price AJ, Beard DJ, Hadfield SG, Arden NK, Murray DW, and Field RE. 2013b.

340 The effects of age on patient-reported outcome measures in total knee replacements.

341 Bone Joint J 95-B:38-44. 10.1302/0301-620X.95B1.28061

342 


\section{Table $\mathbf{1}$ (on next page)}

Age, sex and prognostic scores at baseline among all invited participants and the survey responders. 
1 Table 1. Age, sex and prognostic scores at baseline among all invited participants and the survey 2 responders.

3

\begin{tabular}{|l|c|c|c|}
\hline & $\begin{array}{c}\text { All } \\
(\mathrm{n}=361)\end{array}$ & $\begin{array}{c}\text { Respondents at 6 months } \\
(\mathrm{n}=307)\end{array}$ & $\begin{array}{c}\text { Respondents at 12 months } \\
(\mathrm{n}=303)\end{array}$ \\
\hline Age & $69.2(7.9)$ & $68.6(7.9)$ & $68.8(7.8)$ \\
\hline Female, $\mathrm{n}(\%)$ & $207(57 \%)$ & $176(57 \%)$ & $172(57 \%)$ \\
\hline $\begin{array}{l}\text { PT Prognosis, } \\
\mathrm{n}(\%)\end{array}$ & & & $55(18 \%)$ \\
\hline 10 & $59(16 \%)$ & $54(18 \%)$ & $102(34 \%)$ \\
\hline 9 & $127(35 \%)$ & $109(35 \%)$ & $82(27 \%)$ \\
\hline 8 & $96(27 \%)$ & $81(26 \%)$ & $39(13 \%)$ \\
\hline 7 & $48(13 \%)$ & $37(12 \%)$ & $18(6 \%)$ \\
\hline 6 & $21(6 \%)$ & $17(6 \%)$ & $6(2 \%)$ \\
\hline 5 & $8(2 \%)$ & $7(2 \%)$ & $2(1 \%)$ \\
\hline 4 & $2(1 \%)$ & $2(1 \%)$ & \\
\hline PT, Physiotherapist & \multicolumn{3}{|l}{} \\
\hline
\end{tabular}

4

5 


\section{Table 2 (on next page)}

Group means and standard deviations (SD) in the different outcome measures at the 6and 12-months follow-up. 
1 Table 2. Group means and standard deviations (SD) in the different outcome measures at the 6- and 122 months follow-up.

3

\begin{tabular}{|l|c|}
\hline & Mean (SD) \\
\hline OKS (0-48) & $37.6(7.4)$ \\
6 months & $39.7(7.7)$ \\
12 months & \\
\hline OKS pain (0-100) & $80.2(16.9)$ \\
6 months & $84.7(16.8)$ \\
12 months & $77.4(15.9)$ \\
OKS function (0-100) & $80.0(16.8)$ \\
6 months & $0.843(0.145)$ \\
12 months & $0.868(0.153)$ \\
\hline EQ-5D-3L index (-0.624 to 1.000) & \\
6 months & $78.9(16.7)$ \\
12 months & $79.5(18.3)$ \\
\hline EQ-5D VAS (0-100) & \\
6 months & \\
12 months & \\
\hline OKS: Oxford Knee Score; EQ-5D: EuroQoL 5 \\
Dimensions; VAS: Visual Analog Scale \\
\hline
\end{tabular}

4 
Table 3 (on next page)

Performance of prediction. 
1 Table 3. Performance of prediction.

2

\begin{tabular}{|c|c|c|}
\hline & $\operatorname{AUC}(95 \% \mathrm{CI})$ & $\mathrm{R}^{2}$ \\
\hline \multicolumn{3}{|c|}{ PASS negative answer } \\
\hline 6 months & $0.62(0.54$ to 0.70$)$ & 0.06 \\
\hline 12 months & $0.64(0.56$ to 0.73$)$ & 0.05 \\
\hline \multicolumn{3}{|c|}{ No Treatment Success } \\
\hline 6 months & $0.67(0.59$ to 0.75$)$ & 0.09 \\
\hline 12 months & $0.73(0.65$ to 0.80$)$ & 0.16 \\
\hline
\end{tabular}

3 


\section{Table 4 (on next page)}

Results of the univariate linear regression analyses with the physiotherapists' prognostic scores as predictor 
1 Table 4. Results of the univariate linear regression analyses with the physiotherapists' prognostic scores 2 as predictor

3

\begin{tabular}{|l|c|c|c|c|c|c|}
\hline & \multicolumn{2}{|c|}{6 months } & \multicolumn{2}{c|}{12 months } \\
\hline Dependent variable & Slope $(95 \% \mathrm{CI})$ & $\mathrm{P}$ & $\mathrm{R}^{2}$ & Slope $(95 \% \mathrm{CI})$ & $\mathrm{P}$ & $\mathrm{R}^{2}$ \\
\hline OKS & $1.9(1.2$ to 2.5$)$ & $<0.0001$ & 0.10 & $2.1(1.5$ to 2.8$)$ & $<0.0001$ & 0.12 \\
\hline OKS Pain & $4.0(2.5$ to 5.4$)$ & $<0.0001$ & 0.08 & $4.5(3.0$ to 5.9$)$ & $<0.0001$ & 0.11 \\
\hline OKS Function & $3.9(2.5$ to 5.2$)$ & $<0.0001$ & 0.09 & $4.4(2.9$ to 5.8$)$ & $<0.0001$ & 0.10 \\
\hline EQ-5D-3L Index & $0.03(0.02$ to 0.05$)$ & $<0.0001$ & 0.08 & $0.04(0.03$ to 0.06$)$ & $<0.0001$ & 0.12 \\
\hline EQ-5D VAS & $3.2(1.8$ to 4.7$)$ & $<0.0001$ & 0.06 & $3.6(2.0$ to 5.2$)$ & $<0.0001$ & 0.06 \\
\hline
\end{tabular}

OKS: Oxford Knee Score; EQ-5D: European Quality of Life 5 dimensions; VAS: Visual Analog Scale

4

5 
Figure 1

Study flow chart

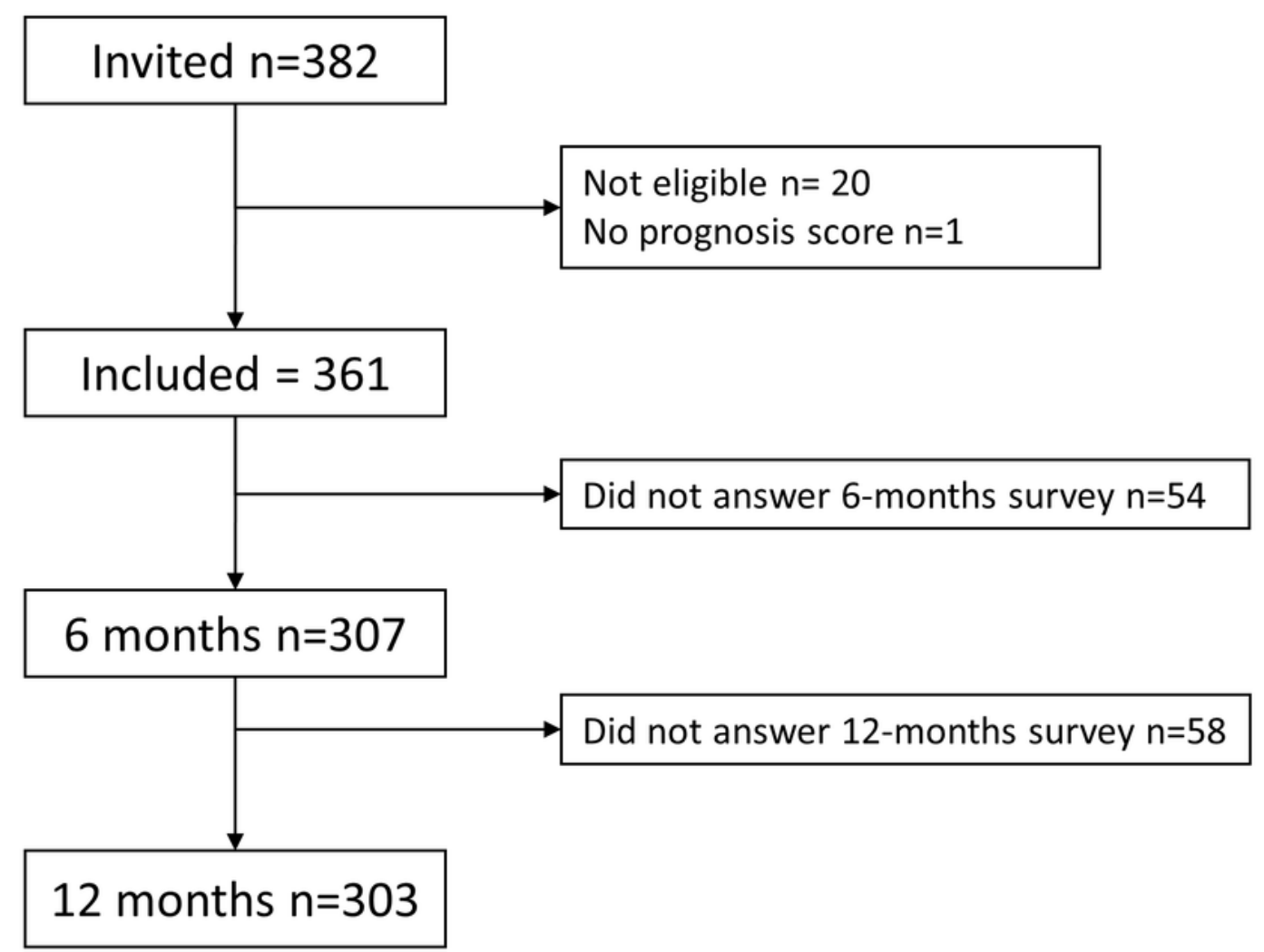

Did not answer any survey: $\mathrm{n}=42$.

Answered 12 months survey but not 6 months survey: $n=12$ Answered 6 months survey but not 12 months survey: $\mathrm{n}=16$ 


\section{Figure 2}

Calibration plots for PASS and Treatment Success at 6 and 12 months.

Predicted probability of achieving either PASS or Treatment Success is given on the x-axis, and the observed probability is given on the $y$-axis. The dashed diagonal line represents perfect agreement between the predicted and actual probability of PASS or Treatment Success. The dots represent the physiotherapists prognostic scores. Error bars are 95\% confidence intervals. At the $x$-axis, the distribution of the predicted probabilities is shown.
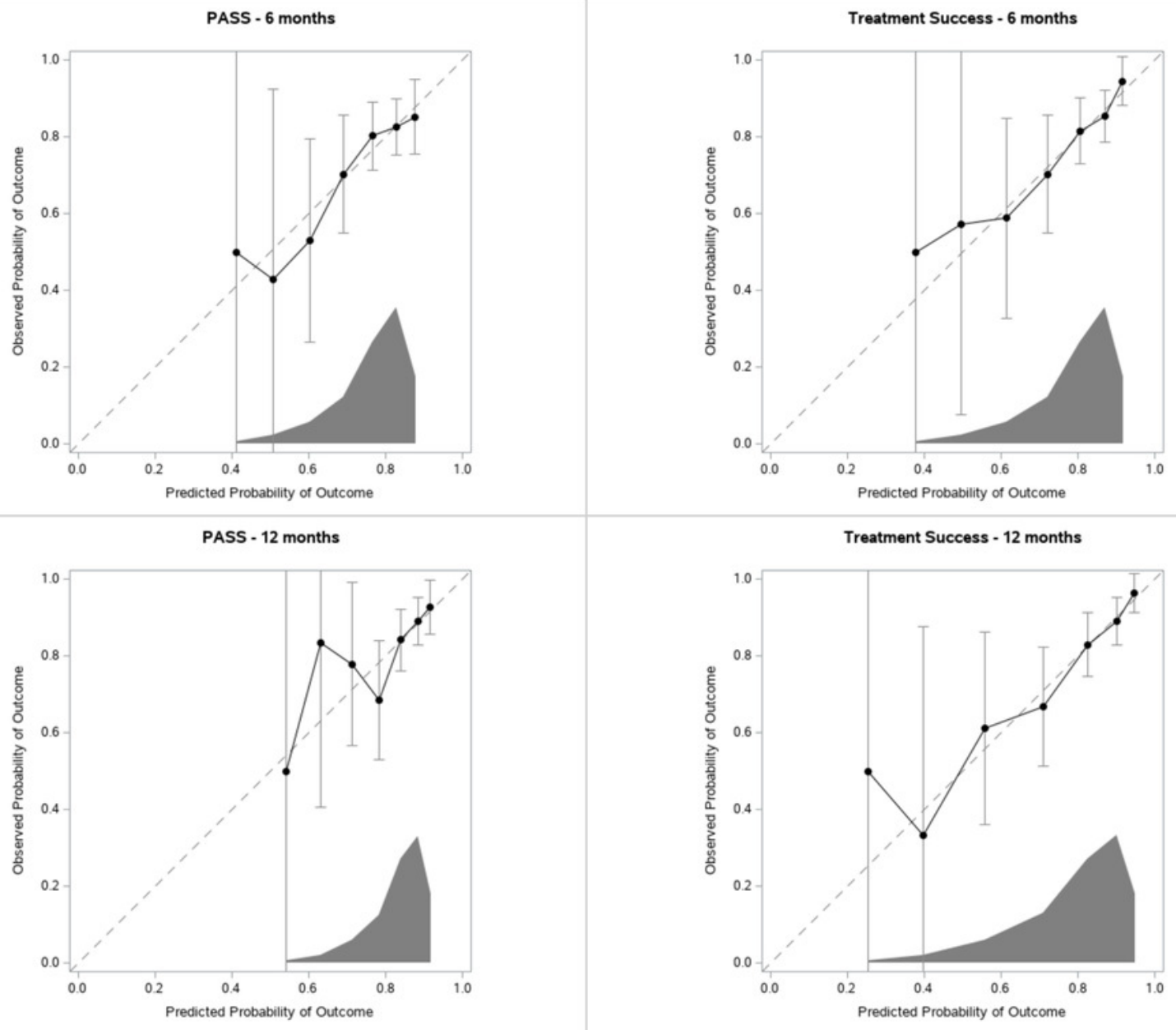